\title{
A new handheld electromagnetic cortical stimulator for brain mapping during open skull neurosurgery: a feasibility study
}

\author{
Jacopo Buzzi ${ }^{1}$, Elena De Momi ${ }^{1}$, Francesco Maria Baratelli ${ }^{2}$, Marco Giacometti ${ }^{2}$ \\ Serena Fiocchi ${ }^{3}$, Marta Parazzini ${ }^{3}$, Paolo Ravazzani ${ }^{3}$, Giancarlo Ferrigno ${ }^{1}$
}

\begin{abstract}
Transcranial magnetic stimulations have provided invaluable tools for investigating nervous system functions in a preoperative context; in this paper we propose an innovative tool to extend the magnetic stimulation to an open skull context as a promising approach to map the brain cortex. The present gold standard for intraoperative functional mapping of the brain cortex, the direct brain stimulation, has a low spatial resolution and limited penetration and focusing capabilities. The magnetic stimulatory device that we present, is designed to overcome these limitations, while working with low currents and voltages. In the present work we propose an early study of feasibility, in which the possibility of exploiting a train of fast changing magnetic fields to reach the neuron's current thresholds is investigated. Measurements of electric field intensity at different distances from the coil, showed that the magnetic stimulator realized is capable of delivering an electric field on a loop of wire theoretically sufficient to evoke neuron's action potential, thus showing the approach' feasibility.
\end{abstract}

\section{STATE OF ART}

A great amount of nowadays neurosurgical procedures take advantage of a detailed both preoperative and intraoperative cortical functional mapping. The first aim of this technique is to understand which are the eloquent areas of the cortex that, if removed, will cause loss of linguistic abilities, paralysis or significant deterioration in the patients quality of life [1] [2] [3] [4]. This is particularly relevant in those surgical procedures that require tissue removal, which is the case of brain tumours treatments or epilepsy surgery, used to remove, or isolate, the seizures foci. In both this procedures the surgeon must remove the greatest part of the area which is considered dangerous while trying to maintain the patient's mobility and ability to talk. Due to the natural anatomical variation between people and the physiologic alteration introduced by the cancer, functionally relevant brain tissue cannot be predicted from anatomical imaging.In the preoperative phase, the most used techniques are the functional magnetic resonance (fMRI) [5] and the transcranial magnetic stimulation (TMS) [6], [7]. The latter uses coil with a radius of usually $5 \mathrm{~cm}$ or more placed on the scalp of the patient used to induce a fast changing magnetic field that is used to induce cortex stimulation. In order to do so though, the TMS uses extremely high currents $(5 \mathrm{kA}$ to $10 \mathrm{kA}$ ) which creates serious safety problems[8] [9]. The

\footnotetext{
${ }^{1}$ Department of Electronics, Informatics and Bioengineering, Politecnico of Milan, Via Ponzio 34/5,20133 Milan, Italy

${ }^{2}$ Biomedical Engineering master students at Politecnico of Milan

${ }^{3}$ Consiglio Nazionale delle Ricerche CNR, Istituto di Elettronica e di Ingegneria dell'Informazione e delle Telecomunicazioni IEIIT, Piazza Leonardo da Vinci 32, 20131, Milan, Italy
}

TMS is often adopted as a rehabilitation technique, with prolonged stimuli and generally lower field intensities [10]. Even though these techniques can noninvasively map the cortex, the preoperative mapping becomes unreliable after the craniotomy, due to the loss of physiological fluids and the consequent brain shift.

For this reason, in this work we will focus on the intraoperative mapping only whose most used procedure, and the first to be developed, is the direct cortical stimulation (DCS) or direct electrical stimulation (DES). In DCS small currents are directly applied on the cortex surface causing small reversible lesions [11], [12].

While DCS is still considered the gold standard for the intraoperative functional mapping, it has major drawbacks. First of all, the direct injection of currents on the brain cortex can induce seizures. These brief and temporary epilepsy events are usually mollified applying cold water directly on the cortex. The seizures are mainly caused by uncontrolled stimulation of the seizure foci with currents that leak unrestrained through the brain tissues. An other main drawback is the resolution of the mapping. Due to the nature of the tools used, which is usually composed of a bipolar stimulator with two electrodes of $1 \mathrm{~mm}$ separated by $5 \mathrm{~mm}$, there are limited capabilities of focusing the current injection. Moreover, with the DCS, the superficial part of the cortex only can be stimulated thus the technique has a very low penetration.

The aim of this work is to propose an alternative tool for the cortical mapping, allowing for a navigated, contactless stimulation of the cortex using small coils inducing fastchanging magnetic field on the brain tissues. The concept is to exploit the TMS technique during the intraoperative phase, using much smaller coils, lowering the current flowing within the spring while maintaining the possibility of focusing the fields in a narrow portion of the cortex.

\section{MATERIAL AND METHODS}

\section{A. Magnetic stimulator technical requirements}

The aim of the device is to induce the activity of the cortex's neurons, in order to do so a sufficient current must be generated. To generate a current on the cortex, a fast time changing magnetic field has to be created. In this study we chose to use a coil as a magnetic field generator. When a current is allowed into a wire wrapped in a spiral, a magnetic field is generated; an approximation of the intensity of the magnetic field can be obtained using the Biot-Savar Law so that the portion of the magnetic field $\mathrm{B}$ along the axes of the 
solenoid generated by a segment of the coil can be expressed as in Equation 1.

$$
\mathbf{d B}(z)=\frac{\mu_{0} R^{2}}{\left(R^{2}+z^{2}\right)^{3 / 2}} N I \frac{d l}{l}
$$

Where $z$ is the distance along the axes from the solenoid, $R$ is the radius of the coil, $I$ is the current flowing in the coil of length $l$ and $d l$ is the small portion of the coil and $\mu_{0}$ indicates the permeability of the vacuum inside the coil; in this applications, in order to have fast changing magnetic fields, the volume inside the coil won't be occupied by a ferromagnetic material. The decay is inversely proportional to the cubic of the distance while directly proportional to the current flowing in the coil. Starting from this evidence, it's possible to understand that due to the craniotomy, the distance between the coil and the area that has to be stimulated can be drastically reduced from around $6 \mathrm{~mm}$ (mean thickness of the human skull [13]) to submillimetrical distances thus allowing for the generation of lower magnetic fields.

From the Eq. 1, integrating over the length $l$ the expression of the magnetic field can be obtained as follows.

$$
B(z)=\frac{\mu_{0} N I}{2 l} \int_{z=0}^{z=l} \frac{R^{2}}{\left(R^{2}+z^{2}\right)^{3 / 2}} d l
$$

Eq. 2 shows the importance of the type and shape of the coil; in fact, the magnetic field is directly proportional to the ratio between the number of turns in the solenoid $(\mathrm{N})$ and and its length (1). Multiple studies [14] have been conducted on the different possible shapes that can be adopted for the TMS and many shapes have been studied in terms of ability to focus the stimulation in a narrow area. Due to the similarity of the TMS approach with the proposal, each consideration remains valid.

In order to induce a current on the cortex, a stationary magnetic field is not sufficient. In fact the electric field (E) that produces an anti-electromotive force is generated by a time varying magnetic field in accord with the MaxwellFaraday equation (Eq. 3).

$$
\nabla \times E=-\frac{\partial B}{\partial t}
$$

Which can also be written in an integral form from the Kelvin Strokes equation (Eq. 4).

$$
\oint_{\partial \Sigma} E d l=-\int_{\Sigma} \frac{\partial B}{\partial t} d A
$$

Where $\Sigma$ is a surface bonded by the $\partial \Sigma$ contour, $d l$ and $d A$ are infinitesimal element of the contour and the surface. This relation leads to an important design constraint: the slew rate of the current flowing in the coil defines the intensity of the induced electric field. From the intensity of the electric field $E$, it's possible to obtain the current density inducted on the cortex $(J)$ using the Kirchhof generalization of the Ohm's law.

In order to activate the neuronal response, thus evoking an action potential, the current density of stimulus must be higher than a threshold [15]. The level of this threshold may vary accordingly with the type of neurons and the duration of the stimuli and ranges from around $100 \mu \mathrm{A} / \mathrm{mm}^{2}$ to around $3000 \mu \mathrm{A} / \mathrm{mm}^{2}$ with a $200 \mu \mathrm{s}$ duration. Instead of considering the current density, it's possible to make the same considerations using current thresholds.

Jankowska at al. in the 1975 [16] demostrated that surface stimulations using currents between $200 \mu \mathrm{A}$ and $1500 \mu \mathrm{A}$ reliably activated the pyramid cells of cat and monkey motor cortex. This current were able to activate, with $200 \mu s$ duration of the stimulus, neurons at $2.4 \mathrm{~mm}$ from the electrode tip. The same study underlined the importance of stimulating the deep layers of the cortex, rather than the surface, in order to maintain lower current thresholds.

\section{B. Setup}

Fig. 1 shows the setup that has been realized. The first component of the setup is a custom build pulse generator (Fig. 1.1). The circuit receives a 5V PWM square signal produced by an Arduino $\mathrm{Uno}^{\mathrm{TM}}$ that triggers a three stage charge pump designed to increase the voltage supply of the circuit up to 60V. The charge pump uses multiple capacitors $(220 \mu \mathrm{F})$ as charge storage in order to obtain higher voltage supply from a $20 \mathrm{~V}$ voltage generator. Arduino is used together with multiple MosFets to control the connection of voltages to the different capacitors while the final stage voltage of $60 \mathrm{~V}$ is charged into a high capacitance capacitor $(6600 \mu F)$. A second Arduino square wave and a InsulatedGate Bipolar Transistor (IGBT) are used to trigger the $60 \mathrm{~V}$ discharge inside the coil 1.3). The system can be depicted

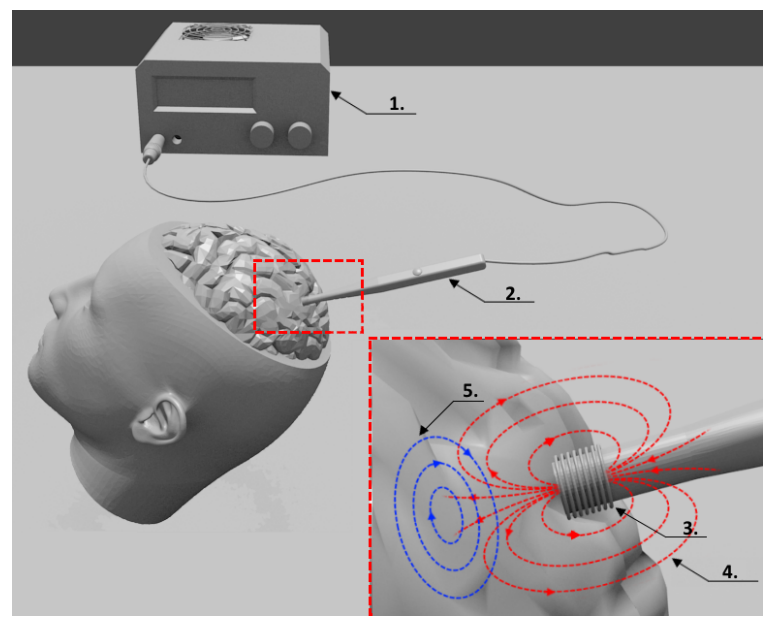

Fig. 1. Setup Model 1. Pulse Generator 2. Stimulator device 3. Coil 4. Magnetic field representation 5. Cortex current representation

with the schema in Fig. 2 that also represent the different signals that are used to produce the pulsating magnetic field. The custom made coil is composed of 4 turns of $0.2 \mathrm{~mm}$ wire wrapped as tight as possible with a inner diameter of the solenoid measuring $7 \mathrm{~mm}$. We have chosen not to use more complex coil shapes in order to limit the number of variables and simplify the data acquisition phase. A resistor in series with the coil has been used to define the maximal current flowing through the system so that, even for very 
low resistance coil, the power consumption and thus the power dissipation requirements can be controlled. In this case we used a $0.6 \Omega$ resistor that allows for theoretical maximal currents higher than $85 \mathrm{~A}$. As can be understood from the previous section, the portions of the $60 \mathrm{~V}$ square wave applied to the coil that can actually induce an electric field are its raise and fall phases. The circuit has been designed such as the maximal slew rate only depends on the discharge constant of the coil-resistor series.

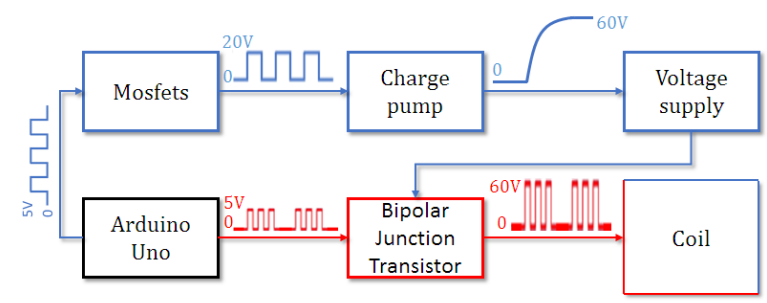

Fig. 2. Schematic model of the system: two square wave $5 \mathrm{v}$ signals from Arduino Uno are used to drive a charge pump, that creates a voltage supply of $60 \mathrm{~V}$, and a IGBT to control the stimulating coil discharge.

Maximising the slew rate of the current inside the coil, the maximal intensity of the magnetic field has been defined. For what concerns the duration of the stimulus, with a fixed slew rate, the transition time during which the current flowing inside the solenoid changes, depends only on the maximal current that can be reached inside the coil-resistor series.

As will be presented in the results, a single stimulus produced by the realized setup last for about $1 \mu \mathrm{s}$ which is well below the stimulation time described in the previous section. In order to take into account this disparity, the induced stimulus is repeated for $2500 \mu \mathrm{s}$ with a square wave frequency of $100 \mathrm{Khz}$. This train is repeated for $2.5 \mathrm{~ms}$ with pauses of $2 \mathrm{~ms}$ between each train. Then the circuit proceed to recharge the voltage supply to $60 \mathrm{~V}$ in around $1 \mathrm{~s}$.

\section{Test Protocol}

In order to understand the real performances of the circuit, the simulated square wave of $60 \mathrm{~V}$ applied to the coil will be compared to the real one, measured on the circuit. The simulation has been obtained with OrCAD pSpice software, modifying already existent components from the public libraries in order to model the actual circuit. The real circuit data has been acquired using the Tektronix TDS3012B $100 \mathrm{MHz} 2$ Channel Oscilloscope and then transmitted to the PC with a crossed Ethernet connection. The choice of using an Oscilloscope instead of a common acquisition board comes from the necessity of acquiring data with high frequency components, around $1 \mathrm{MHz}$.

The coil capability of generating a magnetic field has been tested using a test loop of wire with the same inner diameter as the coil. Using a small resistor, it's possible to acquire the electromotive force induced on the test loop of wire; dividing this value by the length of the loop the electric field can be obtained. Different acquisition of the induced electromotive force obtained on the loop with different separation distance from the coil have been recorded, in order to understand the penetration capability of the system.

In order to have a gross estimation of the actual stimulatory capability of the system, some test have been conducted on the fingertips of different subjects.

\section{RESULTS AND DISCUSSION}

In this section the results obtained validating the magnetic stimulator will be presented. The first graph (Fig. 3) represents the comparison between the simulated single voltage step applied on the coil-resitor series. The two signals appear to be very similar apart from the rising phase. The input square wave from arduino starts at $2 \mu \mathrm{s}$ and ends at $7 \mu \mathrm{s}$; as it can be seen, in the raising phase, both the simulated and the acquired signal waves have a great overshoot peak that reaches $260 \mathrm{~V}$. This behaviour has been obtained using a $20 \Omega$ resistor in series with a high current diode as a discharge circuit for the coil when the IGBT switches from its conductive state to the non conductive one. During this time the current flows inside the $20 \Omega$ resistor rising the potential. The amplitude of this overshoot is directly related with the resistor's resistance value, the inductance of the coil and the time between each stimulus. As will be described in the next results, this peak produces the desired effect of having a differentiation between the positive and negative electric field induced.

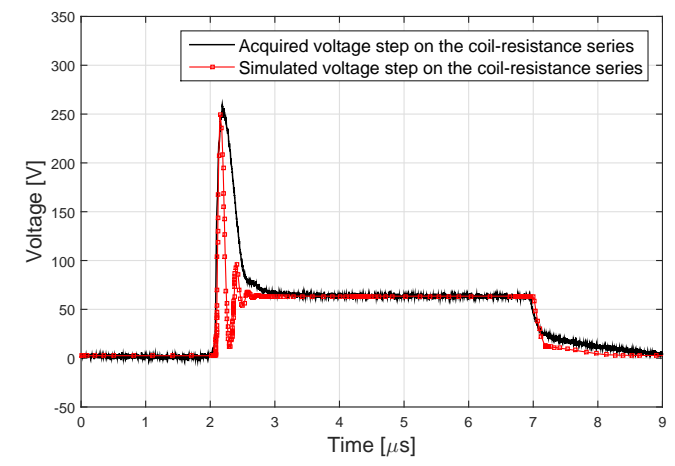

Fig. 3. Comparison between the simulated voltage step on the coil and the acquired one. The voltage overshoot has been obtained exploiting the coil discharge current when the IGBT is not conductive

Fig. 4 shows the comparison between the voltage step applied to the coil and resistor series and the inducted electromotive force recorded with the single loop of wire. In this acquisition the reading loop has been placed perpendicular to the coil axis and its diameter is roughly equal to the stimulation solenoid's one. The coil is able to induce a maximal positive voltage peak of $4.9 \mathrm{~V}$ that lasts for about $1 \mu s$ and it generates a negative peak of $-0.86 \mathrm{~V}$ that last for about $3 \mu \mathrm{s}$. The maximal electric field recorded on the loop is equal to around $200 \mathrm{~V} / \mathrm{m}$

Fig. 5 shows the variation of the inducted electric field as the reading loop distance from the coil is increased. At a distance of $3 \mathrm{~mm}$, which is half of the usual cortex thickness, the maximal electric field recorded is around $100 \mathrm{~V} / \mathrm{m}$ decreasing to $37 \mathrm{~V} / \mathrm{m}$ at $6 \mathrm{~mm}$ from the coil surface. 


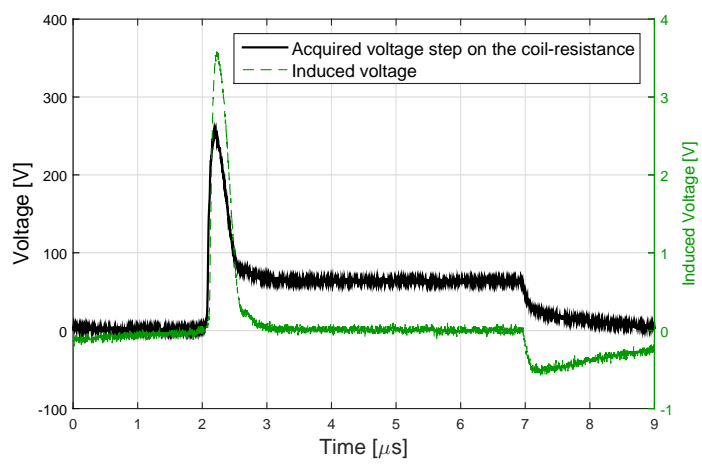

Fig. 4. Comparison between the voltage step applied to the coil and resistor series and the inducted electromotive force recorded with the single loop of wire. Reading loop perpendicular to the coil axis.

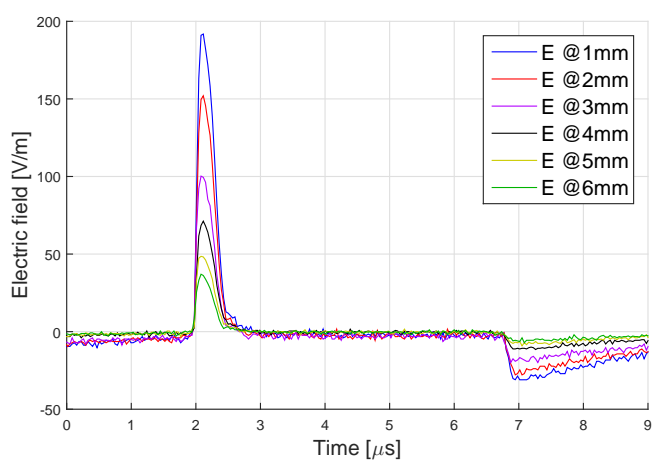

Fig. 5. Comparison between the electric field induced on the same loop of wire at different distances from the coil.

During the qualitative test on different subject's fingertip the stimulation trains where sent at different times and without the subject awareness. Each of the subjects felt a small pressure on the finger-tip during the stimulation but the sensation was very feeble and lasted for a very short amount of time.

\section{CONCLUSION}

In this work the possibilities of building an electromagnetic cortical stimulator for brain mapping during open skull neurosurgery has been studied. This research is inspired by the necessity of obtaining a device that can overcome the DCS limits, thus potentially having a better spatial resolution and thanks to the possibility of focusing the magnetic field, a more controllable penetration in the lower layers of the human cortex.

The actual feasibility of the approach is underlined by the obtained results: the early stimulatory device that has been realized is capable of producing an electric field that can theoretically generate a current density comparable with the neuron's activation threshold while operating with relatively low voltages and currents compared with the TMS.

The future works will focus on those aspects that have been overlooked during this dissertation. First of all an interesting study could be done on the shape and type of the stimulating coil in order to focus the electric field; in this optic the possibility of using multiple coils can also be analysed. Moreover, the timing aspects should be taken into account, thus studying the possibility of having higher currents peaks in order to increase the stimulation time.

\section{REFERENCES}

[1] G. Ojemann, J. Ojemann, E. Lettich, and M. Berger, "Cortical language localization in left, dominant hemisphere," Journal of Neurosurgery, vol. 71, no. 3, pp. 316-326, 1989.

[2] P. G. Matz, C. Cobbs, and M. S. Berger, "Intraoperative cortical mapping as a guide to the surgical resection of gliomas," Journal of Neuro-Oncology, vol. 42, no. 3, pp. 233-245, 1999.

[3] M. M. Haglund, M. S. Berger, M. Shamseldin, E. Lettich, and G. A. Ojemann, "Cortical localization of temporal lobe language sites in patients with gliomas.," Neurosurgery, vol. 34, pp. 567-76; discussion 576, Apr. 1994.

[4] N. Shinoura, A. Midorikawa, R. Yamada, T. Hana, A. Saito, K. Hiromitsu, C. Itoi, S. Saito, and K. Yagi, "Awake craniotomy for brain lesions within and near the primary motor area: A retrospective analysis of factors associated with worsened paresis in 102 consecutive patients.," Surgical neurology international, vol. 4, p. 149, 2013.

[5] A. W. Roe and L. M. Chen, "High-resolution fMRI maps of cortical activation in nonhuman primates: correlation with intrinsic signal optical images.," ILAR journal / National Research Council, Institute of Laboratory Animal Resources, vol. 49, no. 1, pp. 116-123, 2008.

[6] P. Julkunen, L. Säisänen, N. Danner, E. Niskanen, T. Hukkanen, E. Mervaala, and M. Könönen, "Comparison of navigated and nonnavigated transcranial magnetic stimulation for motor cortex mapping, motor threshold and motor evoked potentials," Neuroimage, vol. 44, no. 3, pp. 790-795, 2009.

[7] W. S. Paiva, E. T. Fonoff, M. A. Marcolin, H. N. Cabrera, and M. J. Teixeira, "Cortical mapping with navigated transcranial magnetic stimulation in low-grade glioma surgery.," Neuropsychiatric disease and treatment, vol. 8, pp. 197-201, Jan. 2012.

[8] E. Corthout, A. T. Barker, and A. Cowey, "Transcranial magnetic stimulation. Which part of the current waveform causes the stimulation?,' Experimental brain research, vol. 141, pp. 128-32, Nov. 2001.

[9] J. O'Shea and V. Walsh, "Transcranial magnetic stimulation," Current Biology, vol. 17, pp. R196-R199, Mar. 2007.

[10] S. Rossi and P. M. Rossini, "TMS in cognitive plasticity and the potential for rehabilitation." Trends in cognitive sciences, vol. 8, pp. 273-9, June 2004.

[11] M. a. Nitsche, D. Liebetanz, A. Antal, N. Lang, F. Tergau, and W. Paulus, "Modulation of cortical excitability by weak direct current stimulation-technical, safety and functional aspects.," Supplements to Clinical neurophysiology, vol. 56, pp. 255-276, 2003.

[12] A. Antal, N. Brepohl, C. Poreisz, K. Boros, G. Csifcsak, and W. Paulus, "Transcranial direct current stimulation over somatosensory cortex decreases experimentally induced acute pain perception.," The Clinical journal of pain, vol. 24, no. 1, pp. 56-63, 2008.

[13] S. K. Law, "Thickness and resistivity variations over the upper surface of the human skull.," Brain topography, vol. 6, no. 2, pp. 99-109, 1993.

[14] K. H. Kraus, L. D. Gugino, W. J. Levy, J. Cadwell, and B. J. Roth, "The use of a cap-shaped coil for transcranial magnetic stimulation of the motor cortex.," Journal of Clinical Neurophysiology, vol. 10, no. 3, pp. 353-362, 1993.

[15] E. J. Tehovnik, "Electrical stimulation of neural tissue to evoke behavioral responses," Journal of Neuroscience Methods, vol. 65, no. 1, pp. 1-17, 1996.

[16] E. Jankowska, Y. Padel, and R. Tanaka, "The mode of activation of pyramidal tract cells by intracortical stimuli.," The Journal of physiology, vol. 249, no. 3, pp. 617-636, 1975. 